AUDIT

\title{
Non-attendance at clinic: cycles of audit of a consultant based gastroenterology outpatient department
}

\section{C Bateson}

Postgrad Med J 2004;80:615-616. doi: 10.1136/pgmj.2003.013797

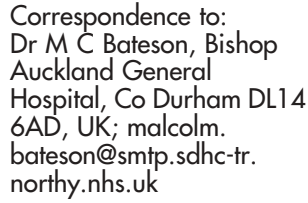

Submitted 20 August 2003 Accepted 27 August 2003

\begin{abstract}
Attempts were made to reduce defaulting rates for new appointments to see a consultant in a general medical and gastroenterology clinic. Despite optimising the operation of the clinic, the non-attendance rate remained unsatisfactory, and comparable to the average for the NHS. There were short term improvements in defaulting rates, which appeared to result from extraneous factors such as a general election and moving to new buildings. Though efforts to improve attendance rates seem appropriate to conserve resources, no definite recommendations can be made on the results of this study.
\end{abstract}

$\mathrm{N}$ ewly referred patients who fail to keep appointments are a conundrum. Defaulting rates are very variable, so it cannot be assumed that a certain proportion of each clinic will not come, permitting routine overbooking. This means that the time allocated to an absent patient is wasted. The official NHS defaulting rate was given as $12 \%$ in 1995 , and is still around this level. One medical and gastrointestinal clinic recorded a defaulting rate of $17 \%,{ }^{1}$ and Aberdeen recorded an overall $22 \%$ non-attendance rate. ${ }^{2}$

\section{STUDIES}

To try and optimise conditions so that there was the best chance of good attendance our local gastroenterology clinic was organised so that all the patients' appointments were made within a month of receipt of the referral letter, usually two to three weeks. Thus patients generally received two to three weeks' notice of appointments. Unique individual appointment times were given and all patients were seen by the consultant. Clinics were not organised when the consultant was absent on holiday or study leave. Punctuality was emphasised so that all patients were seen within 30 minutes of the appointment time, if they arrived on time.

If patients did not attend, the general practitioner was notified accordingly, but routine reappointments were not made. A further appointment required a further formal general practitioner referral. Non-attending patients were not further contacted by questionnaire because it was felt the response rate would be very low and unreliable.

The local population served is approximately 130 000, with a scattered mixed urban and rural community and less than $0.5 \%$ from ethnic minorities. There is little language difficulty.

\section{RESULTS}

Figures were collected and attempts made to explain the failure of some patients to attend. Despite attempts to idealise the service the defaulting rate from May 1994 to December 1996 was $11.3 \%$, not different from the national average.

From December 1995 to February 1996 a system of reminders was instituted. A week before the clinic date, patients were asked by letter to ring to confirm attendance. If they did not do so, they were contacted at home if they were on the telephone. It made absolutely no difference. For the previous six months, May to November 1995, there were 25 out of $253(9.9 \%)$ defaulters, compared with 10 out of 102 $(9.8 \%)$ in the study period. The technique was abandoned as a waste of resources, though seems to have worked elsewhere, ${ }^{3}$ reducing rates in a diabetic clinic from $15 \%$ to $4.6 \%$.

From January 1997, continuous audit of attendance of new patient referrals has been undertaken. No additional measures were taken to improve compliance and there was clearly some spontaneous fluctuation. The overall figure was slightly better than the national average and may reflect the fact that it was known that clinic performance was under scrutiny.

Two extraneous factors did have a profound temporary effect on attendance. The general election of May 1997 was followed by a honeymoon period of five months when defaulting rate fell sharply to $5.6 \%$ (10/178: $p<0.02 v$ May 1994 to April 1997). This effect was then lost and defaulting rates returned to usual levels. Clearly, the change in administration had led the population to expect better things from a restored health service. Interestingly no such change was seen after the 2001 general election, which merely returned the same government.

In the first five months of 2002, the defaulting rate again fell abruptly to $5.3 \%(11 / 209: \mathrm{p}<0.05 v 1998-2001)$. This was probably explained by the construction of a new hospital to replace the World War II emergency medical services building and the associated transfer of service. Not only may patients have been more attracted to a new building, but the date of transfer was initially stated as June, then advanced two months, and then put back to the original date. Most patients during this period received additional correspondence to make clear to which hospital to report and this may have worked as an effective reminder. This improvement was gradually lost in the later months of the year.

\section{Reasons}

Mostly no definite reason could be determined for the nonattendance of patients. When general practitioners were circulated by questionnaire for those absentees with unknown reasons, they only unusually knew the cause. Unlike other studies, administrative failure to send the appointment was rare. 


\begin{tabular}{llllllllll}
\hline Table 1 & \multicolumn{1}{l}{ Clinic non-attendance rates } \\
\hline & 1997 & 1998 & 1999 & 2000 & 2001 & 2002 & Total \\
\hline Total new appointments & 424 & 484 & 464 & 473 & 494 & 494 & 2833 \\
Defaulting rate (\%) & 7.8 & 9.1 & 7.1 & 9.1 & 12.8 & 10.3 & 9.4 \\
\hline
\end{tabular}

In the minority of cases where reasons for non-attendance were definitely known, they fell into three main categories.

\section{Medical}

Death, illness confining to home, admission to hospital, referral elsewhere, and recovery from symptoms were all identified as reasons for not coming.

\section{Behavioural}

Alcoholics, intravenous drug users with hepatitis C, the newly discovered pregnant, and previous or habitual nonattenders were a poorly compliant group. In addition, "urgent" referrals had a tripling of the rate of no-shows, for reasons that could not usually be determined.

\section{Social}

Failure of ambulance or bus transport to arrive, car breaking down, at work, too busy, away on holiday or working away, or mistaken date were all reasons given for not coming by some patients.

\section{DISCUSSION}

It is difficult to influence the non-attending rate for clinic appointments, though it will fluctuate. Routine reappointments are likely to worsen figures, as the chance of a second non-attendance is much higher than that of defaulting on the initial appointment (about 25\%-30\% is the local experience).

\section{Suggestions}

Various methods might be used to improve clinic attendance.

(1) A deposit could be required when the date is made, to be refunded in full if the patient arrives on time. This might be difficult to implement for the poor and old, who may have the system waived or underwritten by the social services department, which would defeat the objective.
(2) Online computer booking might improve compliance, as a patient would know at the time of referral when the clinic appointment was to be. However, routine online booking would circumvent consultants' censoring of referral letters. This might lead to a surge in demand and the appearance of long waiting lists.

(3) The new primary care trusts might be charged with responsibility of ensuring a good attendance of patients referred by general practitioners, with a system of rewards and/or penalties for performances where appropriate.

(4) Patients could be offered evening or weekend clinics to overcome some of the social problems. However, there is no clear evidence this would improve non-attendance rates and such a system would require further careful auditing.

(5) Partial booking may be useful. ${ }^{4}$ Patients are contacted to inform them that a clinic appointment has been requested, and they are then asked to make a date from a range of options. No appointment is made if the patient does not confirm it this way. This has reduced non-attendance rates from $17 \%$ to $5 \%$ in an orthopaedic clinic with a current 53 week wait, ${ }^{5}$ and from $16 \%$ to $5 \%$ in Liverpool, which aims also to offer appointments in four weeks. ${ }^{6}$

\section{REFERENCES}

1 Simmons AV, Atkinson K, Atkinson P, et al. Failure of patients to attend medical out-patient clinic. J R Coll Physicians Lond 1997;31:70-3.

2 Hull AM, Alexander DA, Morrison F, et al. A waste of time: non-attendance at out-patient clinics in a Scottish NHS trust. Health Bulletin 2003;60:62-9

3 Hardy KJ, O'Brien SV, Furlong NJ. Information given to patients before appointments and its effect on non-attendance rate. BMJ 2001;323:1298-300.

4 NHS Modernisation Agency. National booking program. July 2003 (available at: www.modern.nhs.uk).

5 Lloyd J, Dillon D, Hariharan K. Out-patient clinics. Down the line. Health Service Journal 2003; 113:22-3.

6 Scarborough E. Waiting lists. Patients' virtue. Health Service Journal 2002;112:24-5. 